\title{
Apparent Diffusion Coefficient Scalars Correlate with Near- Infrared Spectroscopy Markers of Cerebrovascular Autoregulation in Neonates Cooled for Perinatal Hypoxic- Ischemic Injury
}

A. Tekes, A. Poretti, M.M. Scheurkogel, T.A.G.M. Huisman, J.A. Howlett, E. Alqahtani, J.-H. Lee, C. Parkinson, K. Shapiro, S.-E. Chung, J.M. Jennings, M.M. Gilmore, C.W. Hogue, L.J. Martin, R.C. Koehler, F.J. Northington, and J.K. Lee

\begin{abstract}
BACKGROUND AND PURPOSE: Neurologic morbidity remains high in neonates with perinatal hypoxic-ischemic injury despite therapeutic hypothermia. DTI provides qualitative and quantitative information about the microstructure of the brain, and a near-infrared spectroscopy index can assess cerebrovascular autoregulation. We hypothesized that lower ADC values would correlate with worse autoregulatory function.
\end{abstract}

MATERIALS AND METHODS: Thirty-one neonates with hypoxic-ischemic injury were enrolled. ADC scalars were measured in 27 neonates (age range, 4-15 days) in the anterior and posterior centrum semiovale, basal ganglia, thalamus, posterior limb of the internal capsule, pons, and middle cerebellar peduncle on MRI obtained after completion of therapeutic hypothermia. The blood pressure range of each neonate with the most robust autoregulation was identified by using a near-infrared spectroscopy index. Autoregulatory function was measured by blood pressure deviation below the range with optimal autoregulation.

RESULTS: In neonates who had MRI on day of life $\geq 10$, lower ADC scalars in the posterior centrum semiovale $(r=-0.87, P=.003, n=$ 9) and the posterior limb of the internal capsule $(r=-0.68, P=.04, n=9)$ correlated with blood pressure deviation below the range with optimal autoregulation during hypothermia. Lower ADC scalars in the basal ganglia correlated with worse autoregulation during rewarming $(r=-0.71, P=.05, n=8)$.

CONCLUSIONS: Blood pressure deviation from the optimal autoregulatory range may be an early biomarker of injury in the posterior centrum semiovale, posterior limb of the internal capsule, and basal ganglia. Optimizing blood pressure to support autoregulation may decrease the risk of brain injury in cooled neonates with hypoxic-ischemic injury.

ABBREVIATIONS: $\mathrm{ACS}=$ anterior centrum semiovale; HII = hypoxic-ischemic injury; HVx = hemoglobin volume index; IQR = interquartile ranges; NIRS = near-infrared spectroscopy; MAP = mean arterial blood pressure; MAP $_{\mathrm{OPT}}=$ optimal MAP; PCS = posterior centrum semiovale; $\mathrm{PLIC}=$ posterior limb of the internal capsule; PP = putamen and globus pallidus

$\mathrm{N}$ eonatal hypoxic-ischemic injury (HII) may cause significant and life-long neurologic disabilities. ${ }^{1,2}$ Although therapeutic hypothermia has improved overall neurologic outcomes, death and disability still occur in $30 \%-70 \%$ of affected neonates despite treatment. ${ }^{3-5}$ Early identification of brain injury and risk stratification of neonates with HII are critical for prognostication, adjuvant therapies, family counseling, and additional refinement of hypothermia protocols. Qualitative and quantitative MR imaging serves as an early biomarker of neurologic outcome in HII. ${ }^{5,6}$ Advanced MR imaging techniques such as DTI can detect and quantify abnormalities that may be subtle or under-recognized by conventional MR imaging alone. DTI provides information about the tissue microstructure in vivo by measuring the 3D magnitude, shape, and direction of diffusion of water molecules within the brain. ${ }^{7}$ ADC allows quantification of the magnitude of water dif-

Orleans St, Zayed 4th floor, Room 4155, Baltimore, MD 21205; e-mail: atekes1@jhmi.edu

- Indicates open access to non-subscribers at www.ajnr.org

http://dx.doi.org/10.3174/ajnr.A4083 
fusion within the brain noninvasively. Decreased diffusion (characterized by low ADC values) is a common feature of hypoxicischemic injury in the acute and early subacute phases. ${ }^{8}$

Near-infrared spectroscopy (NIRS) is a noninvasive, bedside monitor that can estimate changes in cerebral blood flow. Ensuring stable cerebral perfusion is critical for preventing secondary brain injury after HII. Cerebrovascular autoregulation maintains steady cerebral blood flow across changes in blood pressure within a specific range of mean arterial blood pressure (MAP). The hemoglobin volume index ( $\mathrm{HVx})$ measures vasoreactivity (which mediates autoregulation) by correlating data derived from NIRS to MAP. ${ }^{9,10}$ The range of MAP with the most robust vasoreactivity and optimized autoregulation is called the "optimal MAP" $\left(\mathrm{MAP}_{\mathrm{OPT}}\right)$ and can be identified with HVx. ${ }^{6,11}$ We hypothesized that lower ADC scalars would correlate with greater blood pressure deviation below $\mathrm{MAP}_{\mathrm{OPT}}$ in neonates with $\mathrm{HII}$ who received therapeutic hypothermia.

\section{MATERIALS AND METHODS}

This study was approved by our institutional review board. Nearterm and term neonates (35 gestational weeks and later) with moderate or severe HII were prospectively enrolled for 27 months, after obtaining written informed consent from the parents. Criteria for HII were based on the clinical trial of hypothermia in HII of the Eunice Kennedy Shriver National Institute of Child Health and Human Development Neonatal Research Network. ${ }^{3}$ All enrolled neonates had moderate-to-severe encephalopathy. The neonates' parents had to speak English or Spanish, the languages available on the consent form. Exclusion criteria were lack of an arterial blood pressure catheter, congenital anomalies that make cooling unsafe, or coagulopathy with active bleeding.

\section{Therapeutic Hypothermia Protocol}

Clinical care was determined by the neonatology team and clinical protocol. Hypothermia was delivered with a whole-body cooling blanket (Mul-T-Blanket Hyper/Hypothermia Blanket and MulT-Pad Temperature Therapy Pad; Gaymar Medi-Therm III; Gaymar Industries, Orchard Park, New York) to maintain a rectal temperature of $33.5 \pm 0.5^{\circ} \mathrm{C}$ for 72 hours. Rewarming was conducted during 6 hours (goal, $0.5^{\circ} \mathrm{C} / \mathrm{h}$ ) to normothermia $\left(36.5^{\circ} \mathrm{C}\right)$. Sedation was provided with opiate infusions and boluses. Hemodynamic goals and the decision of whether to initiate vasopressor or pharmacologic inotropic support were determined by the clinicians. When vasoactive medications were indicated, dopamine was given followed by dobutamine, epinephrine, or milrinone as clinically indicated. Neonates were monitored with full montage electroencephalograms during hypothermia and after rewarming and with continuous amplitude-integrated electroencephalogram monitoring (Brainz BRM3 Monitor or CFM Olympic Brainz Monitor; Natus Medical, San Carlos, California) during hypothermia, rewarming, and the first 6 hours of normothermia. Phenobarbital was given for clinical or subclinical/electrographic seizures. Fosphenytoin, levetiracetam, or topiramate was added for persistent/recurrent seizures. Clinicians could view the regional cerebral oxygen saturation from the NIRS monitor, but not the HVx.

\section{MR Imaging}

MR imaging studies were performed on a $1.5 \mathrm{~T}$ clinical scanner (Avanto; Siemens, Erlangen, Germany) by using a standard neonatal 8-channel head coil. Standard neonatal brain MRI with sagittal T1-weighted, axial T2-weighted, and axial SWI was obtained during normothermia. A single-shot, spin-echo, echo-planar axial DTI sequence with diffusion gradients along 20 noncollinear directions was acquired. An effective high b-value of $1000 \mathrm{~s} / \mathrm{mm}^{2}$ was used for each of the 20 diffusion-encoding directions. An additional measurement without diffusion weighting $(b=0$ $\mathrm{s} / \mathrm{mm}^{2}$ ) was performed. For the acquisition of the DTI data, we used the following parameters: TR, $8500 \mathrm{~ms}$; TE, $86 \mathrm{~ms}$; section thickness, $2.0 \mathrm{~mm}$; FOV , $192 \times 192 \mathrm{~mm}$; and matrix size, $96 \times 96$ (reconstructed as $192 \times 192$ with zero-filled interpolation). A parallel imaging integrated parallel acquisition technique factor 2 with generalized auto-calibrating partial parallel acquisition reconstruction was used. The acquisition was repeated twice to enhance the SNR. ADC maps were automatically calculated by the vendor-specific software in the MR imaging scanner.

Image analysis was performed on the PACS workstation. ADC scalars were measured by region-of-interest analysis on the PACS workstation. ROIs were manually drawn in 7 anatomic regions in each cerebral hemisphere: anterior centrum semiovale (ACS), posterior centrum semiovale (PCS), posterior limb of the internal capsule (PLIC), putamen and globus pallidus (PP), and the entire thalamus, pons, and middle cerebellar peduncle. Additionally, a region of interest was drawn to cover the entire middle pons. To guarantee correct positioning of the ROIs, we correlated ADC maps with the matching fractional anisotropy maps when necessary (eg, PLIC). Otherwise, the remainder of the anatomic areas was identified on the ADC maps (Fig 1). Each region of interest was measured in 3 contiguous axial sections, and the mean of the 3 values was used as the ADC measure for each anatomic region in each cerebral hemisphere. The radiologist was unaware of the patients' HVx, blood pressures, clinical histories, and conventional MR imaging findings.

\section{Autoregulation Monitoring}

Neonatal cerebral oximetry probes (INVOS; Covidien, Mansfield, Massachusetts) were placed bilaterally on the patient's forehead. NIRS signals and arterial blood pressure data from the bedside monitor (Marquette MAC 500; GE Healthcare, Milwaukee, Wisconsin) were synchronously sampled at $100 \mathrm{~Hz}$ and processed with an analog-to-digital converter (DT9804; Data Translation, Marlboro, Massachusetts) by using ICM + software (Cambridge Enterprises, Cambridge, United Kingdom). ${ }^{6,12}$ Signal artifacts in the MAP and NIRS data (eg, arterial line flushes) were manually removed, and data that accounted for $<1 \%$ of the recording period were excluded as an additional measure to remove artifacts. ${ }^{6}$

The HVx was calculated by using a continuous, moving correlation coefficient between MAP and the relative total tissue hemoglobin attenuation, which is a surrogate measure of cerebral blood volume obtained by NIRS., ${ }^{9,10}$ The relative total tissue hemoglobin attenuation is obtained by NIRS by using light with a wavelength of $805 \mathrm{~nm}$. Because this wavelength is isosbestic to both oxygenated and deoxygenated hemoglobin, relative tissue hemoglobin is not affected by changes in cerebral oxygen satura- 


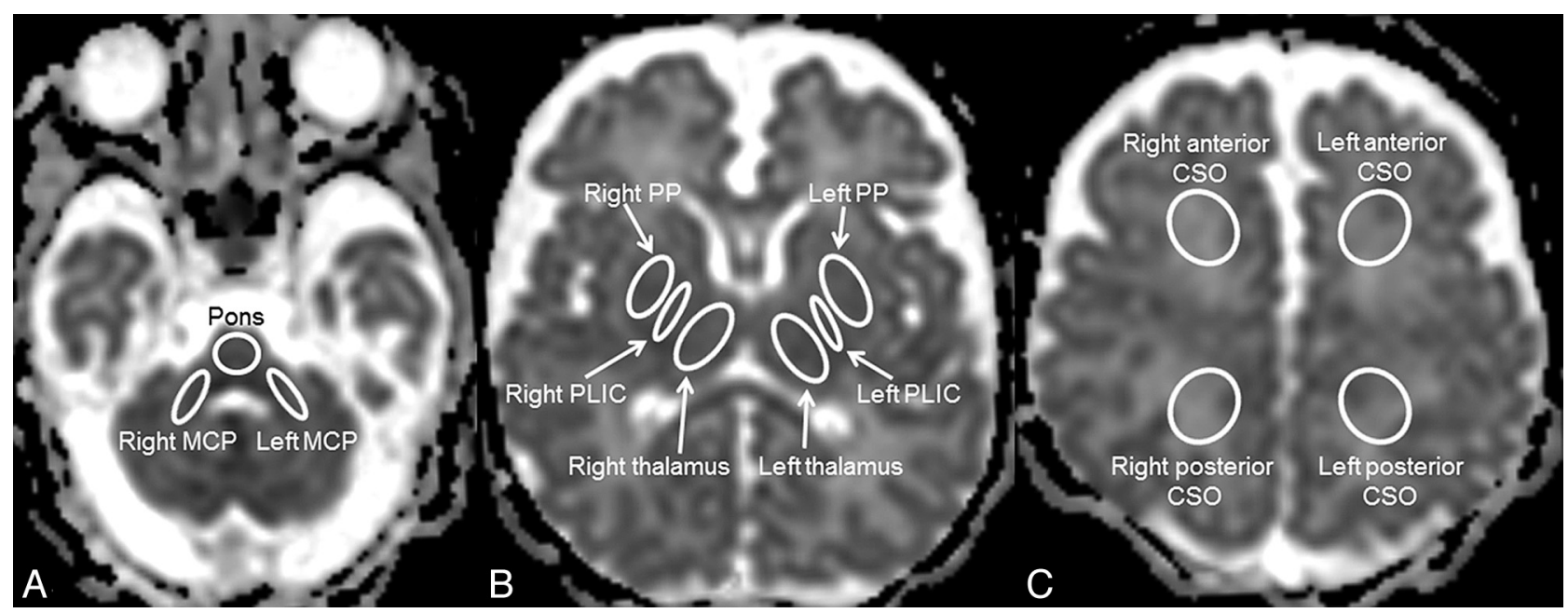

FIG 1. Axial $A D C$ maps at the level of the posterior fossa $(A)$, deep gray matter nuclei $(B)$, and centrum semiovale $(C)$ showing the position of the manually drawn ROls in 7 anatomic regions: the pons $(A), \operatorname{MCPs}(A)$, thalami $(B)$, PLICs $(B)$, PPs $(B)$, and ACS and PCS bilaterally $(C)$. MCP indicates middle cerebellar peduncle

tion. Therefore, HVx can be used to measure autoregulatory vasoreactivity during conditions that affect the relative proportions of oxygenated-to-deoxygenated hemoglobin, such as therapeutic hypothermia (which may affect the cerebral metabolic rate and cardiac output). The use of HVx has been validated in HII piglets undergoing hypothermia and rewarming. ${ }^{10,13}$ Consecutive, paired, 10-second averaged values from a 300-second duration were used for each calculation, thereby incorporating 30 data points for each HVx calculation. ${ }^{12} \mathrm{HVx}$ is a continuous variable that ranges from -1 to +1 . When vasoreactivity is functional (and autoregulation is therefore intact), the cerebral blood volume and MAP either do not correlate or are negatively correlated. This result generates a near-zero or a negative HVx. When vasoreactivity and autoregulation become impaired, the cerebral blood volume and MAP correlate and the HVx becomes positive and approaches $+1 .^{6,9,10}$

Right and left HVx values were averaged and sorted into 5-mm $\mathrm{Hg}$ bins of MAP to generate bar graphs. $\mathrm{MAP}_{\mathrm{OPT}}$ was defined as the bin with the most negative HVx when the graph showed a trend toward increasing index values as MAP deviated from this nadir. ${ }^{6}$ The $\mathrm{MAP}_{\mathrm{OPT}}$ was identified during hypothermia, rewarming, and the first 6 hours of normothermia by 2 physicians independently (J.K.L. and C.W.H.), who were unaware of the patient history and MR imaging results. Both physicians had to agree on the $\mathrm{MAP}_{\mathrm{OPT}}$ to include the neonate in the analysis. Worse autoregulatory function was indicated by spending greater time with blood pressure below $\mathrm{MAP}_{\mathrm{OPT}}$ and greater blood pressure deviation below $\mathrm{MAP}_{\mathrm{OPT}}{ }^{6}$

Autoregulation was analyzed by using 3 methods within each period (hypothermia, rewarming, and the first 6 hours of normothermia). First, the amount of time the neonate spent with blood pressure below $\mathrm{MAP}_{\mathrm{OPT}}$ was calculated. Time with blood pressure below $\mathrm{MAP}_{\mathrm{OPT}}$ was then analyzed as a percentage of the autoregulation monitoring period. Second, the maximum blood pressure deviation below $\mathrm{MAP}_{\mathrm{OPT}}$ was determined. Third, the area under the curve was calculated to combine the amount of time spent with blood pressure below $\mathrm{MAP}_{\mathrm{OPT}}$ and the extent of blood pressure deviation below $\mathrm{MAP}_{\text {OPT. }}$ To cal- culate the area under the curve, we analyzed time as the absolute duration of autoregulation monitoring. The area under the curve (minimum $\times$ millimeter of mercury/hour) for time (minutes) spent with blood pressure below $\mathrm{MAP}_{\mathrm{OPT}}$ and blood pressure deviation (millimeter of mercury) below $\mathrm{MAP}_{\mathrm{OPT}}$ was normalized for monitoring the duration (hours). ${ }^{14}$

\section{Statistical Analysis}

Data were analyzed with SAS, Version 9.2 (SAS Institute, Cary, North Carolina). Graphs were generated with GraphPad Prism (Version 5.03; GraphPad Software, San Diego, California). Data are reported as means with SDs and medians with interquartile ranges (IQRs) when appropriate. A 2-sided $P$ value $\leq .05$ was considered significant. The lowest mean ADC scalar between the right and left cerebral hemispheres was taken as the representative ADC scalar of that anatomic region for the analysis.

ADC scalars in each anatomic region were compared with the percentage of time spent with blood pressure below $\mathrm{MAP}_{\mathrm{OPT}}$, the maximal blood pressure deviation below $\mathrm{MAP}_{\mathrm{OPT}}$, and the area under the curve below $\mathrm{MAP}_{\mathrm{OPT}}$ in each period using Spearman correlations. In addition, the data were stratified for separate analyses of neonates who received their MR imaging before day of life 10 and neonates who received their MR imaging on day of life 10 or later to account for delayed pseudonormalization of ADC in cooled neonates with HII. ${ }^{15}$

\section{RESULTS}

Fifty-six neonates with HII were identified. Twenty-five neonates could not be enrolled in the study due to refusal of consent (8), lack of arterial cannulae (7), withdrawal of care or death soon after birth (4), language barrier (1), known intracranial hemorrhage (1), congenital heart disease (1), extracorporeal membrane support (1), abandonment (1), and inadequate resources (1). Consequently, 31 neonates were enrolled in the study. Autoregulation monitoring could not be accomplished in 1 neonate due to technical problems, and 3 had motion artifacts on MR imaging. Therefore, data were analyzed for 27 neonates ( 12 females and 15 males). 


\section{Apparent Diffusion Coefficient Scalars}

Brain MRI were obtained on median day of life 8 (range, 4-15 days). Mean ADC scalars between the right and left cerebral hemispheres were similar in the PCS $(P=.82), \operatorname{PP}(P=.39)$, PLIC $(P=$ $.47)$, and middle cerebellar peduncle $(P=.35)$. There were differences in mean ADC measures between the right and left hemispheres in the $\operatorname{ACS}(P=.04)$ and thalamus $(P=.04)$.

\section{Autoregulation}

Autoregulation was monitored in 27/27 (100\%) neonates during hypothermia, 25/27 (93\%) during rewarming, and 24/27 (89\%) during normothermia. Reasons for early cessation of monitoring included transfer to the pediatric intensive care unit for extracorporeal membrane support ( 1 neonate) and removal of the arterial blood pressure cannula ( 2 neonates). The median duration of monitoring was 31 hours (IQR, 23-45 hours) during hypothermia, 6 hours (IQR, 5-8 hours) during rewarming, and 6 hours (IQR, 6-6 hours) during normothermia. The neonates' blood pressures are illustrated in Fig 2. $\mathrm{MAP}_{\mathrm{OPT}}$ was identified in 23/27 (85\%) neonates during hypothermia, 24/25 (96\%) during rewarming, and 19/24 (79\%) during normothermia. Median $\mathrm{MAP}_{\mathrm{OPT}}$ values were 50 (IQR, 45-55) during hypothermia, 50 (IQR, 45-55) during rewarming, and 50 (IQR, 45-60) during normothermia.

\section{Apparent Diffusion Coefficient Scalars and Autoregulation}

When all neonates were analyzed together, ADC scalars did not correlate to time spent with blood pressure below $\mathrm{MAP}_{\mathrm{OPT}}$, maximum blood pressure deviation below $\mathrm{MAP}_{\mathrm{OPT}}$, or area under the curve below $\mathrm{MAP}_{\mathrm{OPT}}$ in any period $(P>.08$ during hypothermia $[n=23]$, $P>.07$ during rewarming $[n=24]$, and $P>.10$ during normothermia $[n=19])$. Among neonates who had MRI obtained on day of life $\geq 10$, lower ADC scalars in PCS negatively correlated to time spent with blood pressure below $\mathrm{MAP}_{\mathrm{OPT}}(P<.006)$, blood pressure deviation below $\operatorname{MAP}_{\mathrm{OPT}}(P=.03)$, and area under the curve $(P=$ $.003 ; n=9$ ) during hypothermia. Lower ADC in the PLIC negatively correlated to blood pressure deviation below $\mathrm{MAP}_{\mathrm{OPT}}$ during hypothermia $(P=.04, n=9)$ (Table 1$)$. Lower ADC scalars in PP negatively correlated to blood pressure deviation below $\mathrm{MAP}_{\mathrm{OPT}}$ during rewarming $(P=.05, n=8)$. ADC in the middle cerebellar peduncle correlated to maximal blood pressure deviation below $\mathrm{MAP}_{\mathrm{OPT}}$ during rewarming $(P=.02, n=8)$ (Table 2 ). Blood pressure and ADC did not correlate during normothermia (Table 3). ADC and blood pressure did not correlate during any period among neonates who underwent MRI on day of life $<10$.

\section{DISCUSSION}

Severe neurologic disabilities persist in survivors of HII despite therapeutic hypothermia. ${ }^{1,2} \mathrm{HII}$ is characterized by a period of reduced blood flow (ischemia) and oxygen delivery (hypoxia) followed by reperfusion with transient energy recovery and then secondary energy failure. ${ }^{16}$ Therapeutic hypothermia aims to prevent the events that initiate with reperfusion and culminate in cell death. Although hypothermia was initially thought to decrease both mortality and disability rates, recent long-term outcome studies show significance
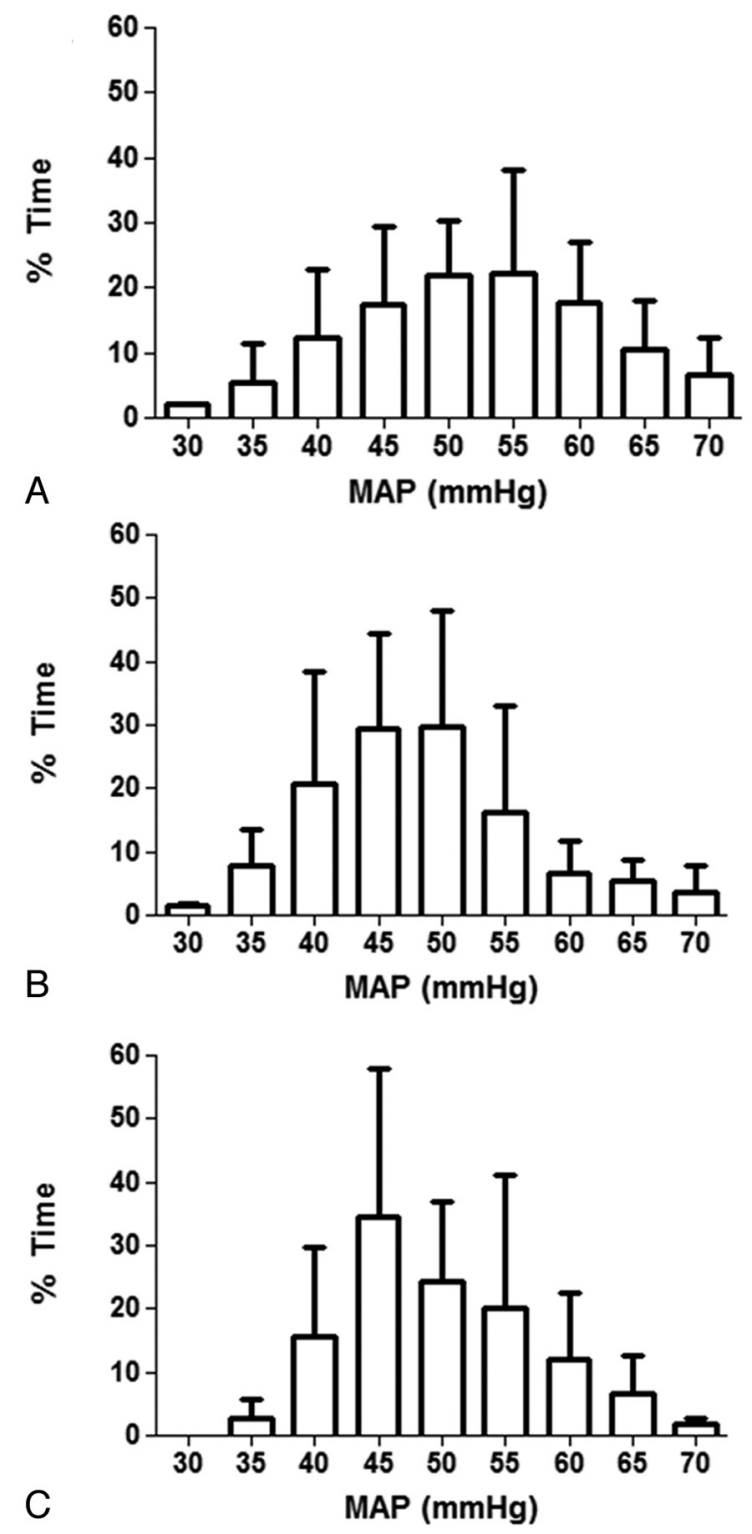

FIG 2. The percentage of the autoregulation monitoring period that neonates spent at each mean arterial blood pressure during hypothermia $(n=27)(A)$, rewarming $(n=25)(B)$, and the first 6 hours of normothermia $(n=24)$ (C). Data are shown as means with SDs. The neonates' blood pressures were similar across time periods.

only for decreased mortality. ${ }^{17,18}$ Research to better define which patients would benefit most from therapeutic hypothermia and the optimal duration of hypothermia is needed. Moreover, neonates who do not fit the current inclusion criteria for HII in clinical trials might still benefit from therapeutic hypothermia. ${ }^{18,19}$ Thus, there is an urgent need to better define the effects of cooling and rewarming on the brain in neonates with HII.

Advanced MR imaging techniques, including DTI and MR spectroscopy, serve as biomarkers of brain injury in HII. ${ }^{20,21}$ However, MR imaging is usually done after the completion of hypothermia and when the child is hemodynamically stable. Autoregulation monitoring with $\mathrm{HVx}$ can be used continuously at the bedside and during hypothermia. By identifying the MAP range that optimizes autoregulation with $\mathrm{HVx}$, clinicians could target neuroprotective hemodynamic goals that reduce the risk of ischemia. 
Table 1: Correlation of ADC scalars and blood pressure in relation to MAP ${ }_{\text {OPT }}$ during therapeutic hypothermia

\begin{tabular}{|c|c|c|c|}
\hline & \multicolumn{3}{|c|}{ Maximum MAP } \\
\hline & $\begin{array}{c}\text { \% Time below } \\
\text { MAP OPT }_{\text {Or }} \\
\text { (P Value) }\end{array}$ & $\begin{array}{c}\text { Deviation below } \\
\text { MAP OPT }(r) \\
(P \text { Value })\end{array}$ & $\begin{array}{l}\text { AUC below } \\
\text { MAP }_{\text {OPT }}(r) \\
\text { (P Value) }\end{array}$ \\
\hline \multicolumn{4}{|c|}{$\mathrm{DOL}<10(n=14)$} \\
\hline ACS & $0.31(.28)$ & $0.36(.20)$ & $0.38(.19)$ \\
\hline PCS & $0.12(.67)$ & $0.25(.39)$ & $0.21(.47)$ \\
\hline PP & $-0.07(.81)$ & $0.06(.84)$ & $-0.04(.90)$ \\
\hline Thalamus & $0.01(.97)$ & $0.13(.67)$ & $0.05(.85)$ \\
\hline PLIC & $0.10(.74)$ & $0.19(.51)$ & $0.18(.55)$ \\
\hline Pons & $0.44(.12)$ & $0.40(.16)$ & $0.45(.11)$ \\
\hline MCP & $-0.32(.27)$ & $-0.10(.74)$ & $-0.25(.39)$ \\
\hline \multicolumn{4}{|c|}{$\mathrm{DOL} \geq 10(n=9)$} \\
\hline ACS & $-0.58(.10)$ & $-0.34(.38)$ & $-0.58(.10)$ \\
\hline PCS & $-0.83(.006)^{b}$ & $-0.71(.03)^{\mathrm{b}}$ & $-0.87(.003)^{\mathrm{b}}$ \\
\hline PP & $-0.47(.20)$ & $-0.19(.62)$ & $-0.35(.36)$ \\
\hline Thalamus & $-0.44(.23)$ & $-0.43(.25)$ & $-0.47(.21)$ \\
\hline PLIC & $-0.61(.08)$ & $-0.68(.04)^{b}$ & $-0.57(.12)$ \\
\hline Pons & $-0.05(.90)$ & $0.07(.86)$ & $0.03(.93)$ \\
\hline MCP & $-0.08(.85)$ & $-0.16(.68)$ & $-0.08(.83)$ \\
\hline
\end{tabular}

Note:-DOL indicates day of life; MCP, middle cerebellar peduncle; AUC, area under the curve.

${ }^{a}$ Autoregulation measurements are the following: the percentage of time spent with blood pressure below MAP ${ }_{\mathrm{OPT}}$, maximal blood pressure deviation below MAP $\mathrm{OPT}_{\text {, }}$ and AUC below MAP $\mathrm{OPT}$. Data were analyzed by Spearman correlations. $\mathrm{b} P \leq .05$.

Table 2: Correlation of ADC scalars and blood pressure in relation to $\mathrm{MAP}_{\mathrm{OPT}}$ during rewarming ${ }^{\mathrm{a}}$

\begin{tabular}{|c|c|c|c|}
\hline & \multicolumn{3}{|c|}{ Maximum MAP } \\
\hline & $\begin{array}{c}\text { \% Time below } \\
\operatorname{MAP}_{\text {OPT }}(r) \\
(P \text { Value })\end{array}$ & $\begin{array}{c}\text { Deviation below } \\
\text { MAP OPT }_{\text {Or })} \\
(P \text { Value })\end{array}$ & $\begin{array}{c}\text { AUC below } \\
\mathrm{MAP}_{\text {OPT }}(r) \\
(P \text { Value })\end{array}$ \\
\hline \multicolumn{4}{|c|}{$\mathrm{DOL}<10(n=16)$} \\
\hline ACS & $-0.25(.36)$ & $-0.06(0.83)$ & $-0.23(0.40)$ \\
\hline PCS & $-0.14(.59)$ & $0.005(.99)$ & $-0.09(.75)$ \\
\hline PP & $-0.02(.93)$ & $-0.10(.71)$ & $-0.07(.79)$ \\
\hline Thalamus & $-0.22(.42)$ & $-0.15(.58)$ & $-0.24(.38)$ \\
\hline PLIC & $-0.22(.41)$ & $0.05(.86)$ & $-0.19(.49)$ \\
\hline Pons & $-0.13(.64)$ & $0.14(.62)$ & $-0.03(.91)$ \\
\hline MCP & $-0.20(.46)$ & $-0.22(.42)$ & $-0.18(.50)$ \\
\hline \multicolumn{4}{|c|}{$\mathrm{DOL} \geq 10(n=8)$} \\
\hline ACS & $-0.55(.16)$ & $-0.18(.68)$ & $-0.45(.26)$ \\
\hline PCS & $-0.21(.61)$ & $0.24(.57)$ & $-0.10(.82)$ \\
\hline $\mathrm{PP}$ & $-0.64(.09)$ & $-0.35(.39)$ & $-0.71(.05)^{\mathrm{b}}$ \\
\hline Thalamus & $0.24(.57)$ & $0.42(.31)$ & $-0.10(.82)$ \\
\hline PLIC & $0.14(.74)$ & 0 & $-0.05(.91)$ \\
\hline Pons & $-0.14(.74)$ & $0.01(.98)$ & $0.17(.69)$ \\
\hline MCP & $0.57(.14)$ & $0.79(.02)^{b}$ & $-0.48(.23)$ \\
\hline
\end{tabular}

Note:-DOL indicates day of life; MCP, middle cerebellar peduncle; AUC, area under the curve.

${ }^{a}$ Autoregulation measurements are the following: the percentage of time spent with blood pressure below $\mathrm{MAP}_{\mathrm{OPT}}$, maximal blood pressure deviation below $\mathrm{MAP}_{\mathrm{OPT}}$,

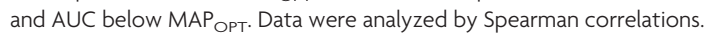
${ }^{\mathrm{b}} \mathrm{P} \leq .05$.

In our study, lower ADC scalars in the PCS, PLIC, and PP correlated with blood pressure deviation below $\mathrm{MAP}_{\mathrm{OPT}}$ during hypothermia and rewarming in neonates who had MRI on day of life $\geq 10$. An association between qualitative MR imaging measures of injury in the PP and thalamus and blood pressure below $\mathrm{MAP}_{\mathrm{OPT}}$ during rewarming was previously reported in HII. ${ }^{6}$ Here, we identified additional correlations between hypoxic-ischemic injury to the PCS and PLIC and blood pressure autoregulation. This reflects the added value of quantitative $\mathrm{ADC}$ measurements as opposed to qualitative/
Table 3: Correlation of ADC scalars and blood pressure in relation to MAP $_{\text {OPT }}$ during the first 6 hours of normothermia ${ }^{a}$

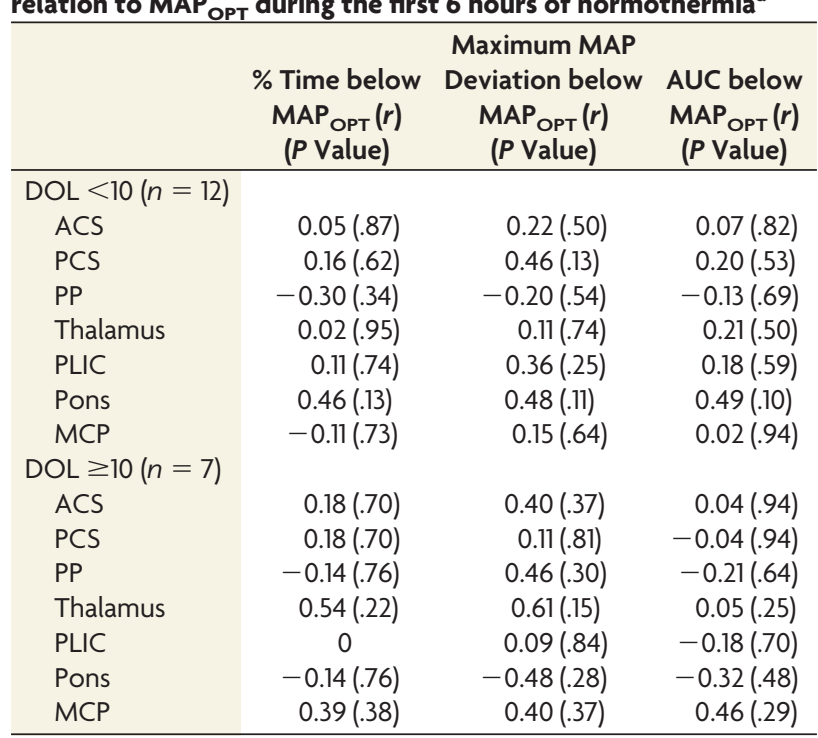

Note:-DOL indicates day of life; MCP, middle cerebellar peduncle; AUC, area under the curve.

${ }^{a}$ Autoregulation measurements are the following: the percentage of time spent with blood pressure below $\mathrm{MAP}_{\mathrm{OPT}}$, maximal blood pressure deviation below MAP $\mathrm{OPT}_{\mathrm{OP}}$

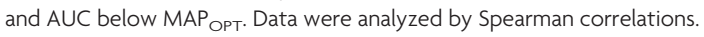

subjective MR imaging analysis alone. Thalamic ADC scalars did not correlate with HVx in this study, possibly because the entire thalamus was included in our region of interest and we did not have the precision to delineate subcompartments of the thalamus that show differential vulnerability to HII.

Correlation between lower ADC scalars and worse autoregulation was observed in the PCS but not in the ACS. The absence of an association between ADC scalars in ACS and autoregulation is surprising and could reflect differences in the effects of HII on the anterior-versus-posterior circulations. In the posterior fossa, no correlation was observed in the pons, but a positive correlation between $\mathrm{ADC}$ and blood pressure occurred in the middle cerebellar peduncle. Measures of autoregulation obtained over the frontal cortex may not be relevant to ischemia in the posterior fossa. These findings emphasize the complex relationships among HII, regional ischemic injury, and autoregulation measurements by using NIRS technology.

The temporal cascade of events that take place during HII is quite complex, and the impact of cooling adds another piece to this puzzle. Although we had a variation in timing of brain MR imaging, this variation enabled us to factor in delayed pseudonormalization in our analysis. ADC scalars and blood pressure only correlated in neonates who received MRI on day of life $\geq 10$. Pseudonormalization of ADC values is delayed in cooled neonates through 10 days of age. ${ }^{15}$ Our study included neonates with moderate or severe HII, and we did not further score the degree of disease severity. Future studies are needed to determine whether more severely injured neonates may have more or less delay in ADC pseudonormalization.

The lowest mean ADC scalar from the right or left cerebral hemisphere was used as the representative measure for that anatomic region. With the exception of the ACS and thalamus, ADC values were similar between the right and left sides in all anatomic regions, including the PCS, PLIC, and PP, where significant correlations between $\mathrm{ADC}$ and blood pressure autoregulation were identified. ADC scalars in the ACS and thalamus did not correlate to blood pressure. 
Therefore, using the lowest ADC value between hemispheres to assess regional ischemic injury was a reasonable approach.

HVx assesses autoregulatory vasoreactivity by measuring total tissue hemoglobin attenuation in the superficial frontal cortex. It does not directly measure deep brain regions. Techniques to continuously measure cerebral blood flow autoregulation in deep regions of the brain are not clinically available for neonates. Whether vascular responses to changes in blood pressure are different in deep brain regions compared with the cortex after global HII and therapeutic hypothermia remains unclear. Nonetheless, the association between $\mathrm{MAP}_{\mathrm{OPT}}$ and ischemia measured by ADC in the PCS, PLIC, and PP suggests that superficial autoregulation measurements may identify blood pressure goals that protect deeper brain regions, as well.

There were several limitations in this study. The sample size was small. The severity of ischemic brain injury or degree of encephalopathy could not be controlled for in the analysis. Whether maintaining blood pressure close to $\mathrm{MAP}_{\mathrm{OPT}}$ to optimize autoregulation decreases the risk of brain injury cannot be determined in this observational study. The blood pressure and autoregulation measurements were obtained during the neonates' first few days of life during hypothermia, rewarming, and the beginning of normothermia, whereas the brain MRI was obtained after normothermia was attained. Moreover, autoregulation monitoring could only begin once an arterial blood pressure cannula was placed and informed consent was obtained. Therefore, disturbances in autoregulation that may have occurred before the start of monitoring were not accounted for in the analysis.

\section{CONCLUSIONS}

This study identified several findings relevant to the treatment of HII. In neonates cooled for HII, lower ADC scalars in the PCS, PLIC, and PP correlated to greater blood pressure deviation below the range with optimal autoregulation. It remains unclear to what extent the ADC changes are attributable to the primary hypoxic ischemic injury versus secondary impaired autoregulation, or a combination of all complex events that take place during the injury and therapeutic hypothermia. Although a causal relationship could not be identified in this observational study, these data suggest that minimizing blood pressure deviation below $\mathrm{MAP}_{\mathrm{OPT}}$ may minimize ischemic injury in these brain regions.

Disclosures: Maureen M. Gilmore—UNRELATED: Grants/Grants Pending: Covidien,* Comments: Covidien previously provided grant funding to Jennifer Lee, MD, the primary investigator leading our group in the autoregulation studies we perform with term and near-term babies with hypoxic-ischemic encephalopathy; Payment for Development of Educational Presentations: eNeonatal Review, Comments: I received an honorarium as the Course Director for this on-line educational literature review of neonatology-related topics. Charles W. Hogue-RELATED: Grant: National Institutes of Health RO1 HL 092259*; UNRELATED: Consultancy: CLS Behring (DSMB), Ornim (Advisory Board), Covidien (served as advisor, but not in past 12 months); Expert Testimony: Dr Hogue has consulted on legal matters, serving as an expert witness in medical litigation in September 2013; Grants/Grants Pending: Covidien.* Raymond C. Koehler-RELATED: Grant: National Institutes of Health. ${ }^{*}$ Jennifer K. Lee-RELATED: Grant: Covidien, ${ }^{*}$ Thomas Wilson Sanitarium for Children, research grant, ${ }^{*}$ American Heart Association*; UNRELATED: Grants/Grants Pending: Covidien.* Frances J. Northington—RELATED: Grant: National Institutes of Health-Eunice Kennedy Shriver National Institute of Child Health and Human Development,* Covidien*; UNRELATED: Grants/Grants Pending: National Institutes of Health-Eunice Kennedy Shriver National Institute of Child Health and Human Development, ${ }^{*}$ Covidien*; Payment for Lectures (including service on Speakers Bureaus): American Academy of Pediatrics (honorarium); Travel/Accommodations/
Meeting Expenses Unrelated to Activities Listed: American Academy of Pediatrics (accommodations and travel), National Institutes of Health (travel to study section). *Money paid to the institution.

\section{REFERENCES}

1. Dilenge ME, Majnemer A, Shevell MI. Long-term developmental outcome of asphyxiated term neonates. J Child Neurol 2001;16:781-92

2. Nelson KB. Neonatal encephalopathy: etiology and outcome. Dev Med Child Neurol 2005;47:292

3. Shankaran S, Laptook AR, Ehrenkranz RA, et al. Whole-body hypothermia for neonates with hypoxic-ischemic encephalopathy. N Engl J Med 2005;353:1574-84

4. Gluckman PD, Wyatt JS, Azzopardi D, et al. Selective head cooling with mild systemic hypothermia after neonatal encephalopathy: multicentre randomised trial. Lancet 2005;365:663-70

5. Azzopardi D, Strohm B, Edwards AD, et al. Treatment of asphyxiated newborns with moderate hypothermia in routine clinical practice: how cooling is managed in the UK outside a clinical trial. Arch Dis Child Fetal Neonatal Ed 2009;94:F260-64

6. Howlett JA, Northington FJ, Gilmore MM, et al. Cerebrovascular autoregulation and neurologic injury in neonatal hypoxic-ischemic encephalopathy. Pediatr Res 2013;74:525-35

7. Poretti A, Meoded A, Rossi A, et al. Diffusion tensor imaging and fiber tractography in brain malformations. Pediatr Radiol 2013;43:28-54

8. Brissaud O, Amirault M, Villega F, et al. Efficiency of fractional anisotropy and apparent diffusion coefficient on diffusion tensor imaging in prognosis of neonates with hypoxic-ischemic encephalopathy: a methodologic prospective pilot study. AJNR Am J Neuroradiol 2010;31:282-87

9. Lee JK, Kibler KK, Benni PB, et al. Cerebrovascular reactivity measured by near-infrared spectroscopy. Stroke 2009;40:1820-26

10. Lee JK, Brady KM, Mytar JO, et al. Cerebral blood flow and cerebrovascular autoregulation in a swine model of pediatric cardiac arrest and hypothermia. Crit Care Med 2011;39:2337-45

11. Zweifel C, Castellani G, Czosnyka M, et al. Noninvasive monitoring of cerebrovascular reactivity with near infrared spectroscopy in head-injured patients. J Neurotrauma 2010;27:1951-58

12. Brady K, Joshi B, Zweifel C, et al. Real-time continuous monitoring of cerebral blood flow autoregulation using near-infrared spectroscopy in patients undergoing cardiopulmonary bypass. Stroke 2010;41:1951-56

13. Larson AC, Jamrogowicz JL, Kulikowicz E, et al. Cerebrovascular autoregulation after rewarming from hypothermia in a neonatal swine model of asphyxic brain injury. J Appl Physiol (1985) 2013;115:1433-42

14. Aronson S, Dyke CM, Levy JH, et al. Does perioperative systolic blood pressure variability predict mortality after cardiac surgery? An exploratory analysis of the ECLIPSE trials. Anesth Analg 2011;113:19-30

15. Bednarek N, Mathur A, Inder T, et al. Impact of therapeutic hypothermia on MRI diffusion changes in neonatal encephalopathy. Neurology 2012;78:1420-27

16. Ferriero DM. Neonatal brain injury. N Engl J Med 2004;351:1985-95

17. Shankaran S, Pappas A, McDonald SA, et al. Childhood outcomes after hypothermia for neonatal encephalopathy. $N$ Engl J Med 2012;366:2085-92

18. Jacobs SE, Berg M, Hunt R, et al. Cooling for newborns with hypoxic ischaemic encephalopathy. Cochrane Database Syst Rev 2013;1: CD003311

19. Gancia P, Pomero G. Therapeutic hypothermia in the prevention of hypoxic-ischaemic encephalopathy: new categories to be enrolled.J Matern Fetal Neonatal Med 2012;25(suppl 4):94-96

20. Bonifacio SL, Saporta A, Glass HC, et al. Therapeutic hypothermia for neonatal encephalopathy results in improved microstructure and metabolism in the deep gray nuclei. AJNR Am J Neuroradiol 2012;33:2050-55

21. Massaro AN, Jeromin A, Kadom N, et al. Serum biomarkers of MRI brain injury in neonatal hypoxic ischemic encephalopathy treated with whole-body hypothermia: a pilot study. Pediatr Crit Care Med 2013;14:310-17 